\title{
Euler-Maruyama Approximation for Mean-Reverting Regime Switching CEV Process
}

\author{
Ruxing $\mathrm{Xu}^{*}$ and Dan $\mathrm{Wu}$ \\ Department of Mathematics, China Jiliang University, Hangzhou, China \\ ${ }^{*}$ Corresponding author
}

\begin{abstract}
The mean-reverting constant elasticity of variance (CEV) process with regime switching is one of the most successful continuous-time models of the short term rate, volatility, and other financial quantities. However, most SDEs with Markovian switching do not have explicit solutions. This paper obtains the Euler-Maruyama approximate solution for mean-reverting Regime Switching CEV processes and provides a detailed proof of the convergence of the EM approximate solution to the exact solution.
\end{abstract}

Keywords-CEV process; mean-reverting; regime switching; Euler-Maruyama; Lipschitz condition

\section{INTRODUCTION}

Option pricing is one of the most important research fields in financial economics from both practical and theoretical point of view. The work of Black and Scholes [1] and Merton [2] laid the foundations of the research field and motivated important research in option pricing theory, its mathematical models and its computational techniques. The Black-ScholesMerton formula is one of the important products of economic research of the last century and it has been widely adopted by traders, analysts , investors and other finance researcher.

Despite its popularity, the Black-Scholes-Merton formula is not without flaws. It has been documented in many studies in empirical finance that the Geometric Brownian Motion (GBM) assumed in the Black-Scholes- Merton model does not provide a realistic description for the behavior of asset price dynamics. One of substitutes is the CEV model, which is originally introduced by Cox [3] and Cox and Ross [4]. Many empirical studies have been conducted in the literature to justify the use of the CEV model, for instance, MendozaArriaga and Linetsky [5], Ruas, Dias and Nunes [6], Larguinho, Dias and Braumann [7], Thakoor, Tangman and Bhuruth [8].

Markovian regime-switching models have drawn a significant amount of attention in recent years due to their ability to include the influence of macroeconomic factors on individual asset price dynamics ${ }^{[9-13]}$. There are substantial empirical evidences in support of the existence of regime switching effects on stock market returns and default probabilities. Using the CRSP stock market returns over the period 1929-1989, Schaller and Norden [14] demonstrate that there is compelling evidence of regime switching in US stock market returns and the evidence for switching is robust to different specifications such as switching in means, switching in variances, and switching in both means and variances. Ang and Timmermann [15] also show that regime-switching models can capture the stylized behavior of many asset returns.

In this paper, we investigate the Euler-Maruyama approximate solution of a stochastic differential equation, where we generalize the mean-reverting CEV process by replacing the constant parameters with the corresponding parameters modulated by a continuous-time, finite-state, Markov chain. This paper obtain the Euler-Maruyama approximate solution for mean-reverting Regime Switching CEV processes and provides a detailed proof of the strong convergence of the EM approximate solution to the exact solution.

This paper is organized as follows. In Section II, we develop a mean-reverting CEV process with regime switching. The Euler-Maruyama approximate solution is provided in Section III. In Section IV, we provide a detailed proof of the strong convergence of the EM approximate solution to the exact solution. Conclusion is given in Section V.

\section{MEAN-REVERTING REGIME SWITCHING CEV PROCESS}

We let $\left(\Omega, F,\{F\}_{t \geq 0}, P\right)$ be a complete probability space with a filtration $F_{t>0}$ satisfying the usual conditions, upon which all stochastic processes are defined. Let $X(t)$ be a finite-state continuous-time Markov chain taking values among $G$ different states, where $G$ is the total number of states considered in the economy. Each state represents a particular regime and is labeled by an integer $i$ between 1 and $G$. Hence the state space of $X(t)$ is given by $\Im:=\{1,2, \cdots, G\}$ which can be used to model factors of the economy. Here the Markov chain is assumed to be observable and serve as a proxy for some exogenous economic factors such as GDP and stock price indices. One might interpret the states of $X(t)$ as different stages of a business cycle. By interpreting the states of the Markov chain $X(t)$ as different stages of a business cycle, one could suppose that $G=5$ and that state 1 , state 2, $\cdots$, and state 5 represent expansion, peak, $\cdots$, and recovery, respectively.

To obtain the transition probabilities of the Markov chain $X(t)$, we need to specify its generator matrix $Q$. For easy 
exposition, we assume that a constant generator $Q=\left(q_{i j}\right)_{G \times G}$ is given. Clearly it is straightforward to extend the framework to the case of time varying generator. From Markov chain theory, the elements $\left(q_{i j}\right)_{G \times G}$ in the matrix Q satisfy:

1. $q_{i j} \geq 0$ if $i \neq j$;

2. $q_{i i} \leq 0$ and $q_{i i}=-\sum_{j \neq i} q_{i j}$ for each $i=1, \cdots, G$.

Assume that the Markov chain $X(t)$ at any time $t>0$ is in a regime $i \in \Im$. Then after a period of time $\Delta t$, the Markov chain $Y_{t+\Delta t}$ may stay in regime $i$ with probability $P^{Y}(i, i)$ or jump to any other regime $j \in \Im$ with probability $P^{X}(i, j)$, where the one-step transition probabilities $P^{X}(i, j)$ of the Markov chain $X(t)$ are given by

$$
p^{x}(i, j)=\left\{\begin{array}{cc}
e^{q_{i i} \Delta t}, & j=i \\
\left(1-e^{q_{i i} \Delta t}\right) \frac{q_{i j}}{-q_{i i}}, & j \neq i
\end{array} .\right.
$$

Let $W(t)$ be a standard Brownian motion defined on the probability space $\left(\Omega, F,\{F\}_{t \geq 0}, P\right)$. We consider the following regime-switching mean-reverting $\mathrm{CEV}$ process

$$
d Y(t)=a_{X(t)}\left(b_{X(t)}-Y(t)\right) d t+\sigma_{X(t)} Y(t)^{\beta} d W(t), t \geq 0,
$$

with initial values $Y_{0}=y_{0}$ and $X_{0}=x_{0} . Y(t)$ represents an underlying variable (for example, the stochastic interest rate or default intensity), $a_{Y(t)}$ denotes the speed of mean reversion, $b_{Y(t)}$ denotes the long term mean of the variable, and $\sigma_{Y(t)}$ is the volatility coefficient. $a_{Y(t)}, b_{Y(t)}$ and $\sigma_{Y(t)}$ are positive real numbe dependent on the Markov chain $Y(t)$, indicating that they can take different values in different regimes.

Since the underlying variable $Y(t)$ is mainly used to model stochastic volatility or interest rate or an asset price, it is critical that $Y(t)$ will never become negative. Mao et al. [10] discuss its analytical properties when $\frac{1}{2} \leq \beta \leq 1$ and show that for given any initial data $Y_{0}=y_{0}>0$ and $X_{0}=x_{0} \in \Im$, the solution $Y(t)$ of (1) will remain positive with probability 1 , namely $Y(t)>0$ for all $t \geq 0$ almost surely, if one of the following two conditions holds:

1. $\frac{1}{2}<\beta \leq 1$;

2. $\beta=\frac{1}{2}$ and $\sigma_{i}^{2} \leq 2 a_{i} b_{i}$ for all $i \in \Im$.

\section{A. Lemma 1.}

The coefficients of (1) satisfy the local Lipschitz condition for given initial value $Y_{0}=y_{0}>0$, i.e., for every integer $k>1$, there exists a positive constant $L_{k}$ such that for all $i \in \Im$, and those $x, y$ with $x \in[0, k]$ and $y \in[0, k]$, and

$\left|a_{i}\left(b_{i}-x\right)-a_{i}\left(b_{i}-y\right)\right| \leq L_{k}|x-y|,\left|\sigma_{i} x^{\beta}-\sigma_{i} y^{\beta}\right| \leq L_{k}|x-y|$,

and thus there exists a unique local solution to equation (1).

The following theorem reveals the existence of the positive solution.

\section{B. Lemma 2}

For any given initial value $Y_{0}=y_{0}>0, a_{i}, b_{i}$ and $\sigma_{i}>0$ for all $i \in \Im$, there exists a unique positive global solution $Y(t)$ to Eq. (1) on $t \geq 0$.

\section{THE EULER-MARUYAMA APPROXIMATE SOLUTION}

To define the Euler-Maruyama approximate solution, we will need the following lemma.

\section{Lemma 3.}

Given a step size $\Delta t>0$, let $x_{k}=X_{k \Delta t}$ for $k=0,1,2$, $\cdots$. Then $x_{k}$ is a discrete-time Markov chain with the onestep transition probability matrix

$$
p^{x}(i, j)=\left\{\begin{array}{cc}
e^{q_{i i} \Delta t}, & j=i \\
\left(1-e^{q_{i i} \Delta t}\right) \frac{q_{i j}}{-q_{i i}}, & j \neq i
\end{array} .\right.
$$

Then the discrete-time Markov chain $x_{k}$ can be simulated as follows:

1. Compute the transition-probability matrix $P^{x}(i, j)$;

2. Let $\widetilde{X_{0}}=i_{0}$ and generate a random number $\xi_{1}$ which is uniformly distributed in $[0,1]$. Let $i_{1} \neq G$ and define

$$
\widetilde{X_{1}}=\left\{\begin{array}{cc}
i_{1}, & \sum_{j=1}^{i_{1}-1} p^{\widetilde{X}}\left(i_{0}, j\right) \leq \xi_{1}<\sum_{j=1}^{i_{1}-1} p^{\widetilde{X}}\left(i_{0}, j\right) \\
G, \quad \sum_{j=1}^{G-1} p^{\widetilde{X}}\left(i_{0}, j\right) \leq \xi_{1}
\end{array}\right.
$$

3. Generate independently a new random number $\xi_{2}$ which is again uniformly distributed in $[0,1]$ and then Let $i_{2} \neq G$ and define

$\widetilde{X_{2}}=\left\{\begin{array}{cc}i_{2}, & \sum_{j=1}^{i_{2}-1} p^{\widetilde{X}}\left(i_{1}, j\right) \leq \xi_{1}<\sum_{j=1}^{i_{2}-1} p^{\widetilde{X}}\left(i_{1}, j\right) \\ G, & \sum_{j=1}^{G-1} p^{\widetilde{X}}\left(i_{1}, j\right) \leq \xi_{2}\end{array}\right.$

4. Repeating this procedure, a trajectory of $\widetilde{X}_{k}, k=0$, $1,2, \cdots$ can be generated. This procedure can be carried out independently to obtain more trajectories. 
After explaining how to simulate the discrete-time Markov chain $\widetilde{X_{k}}$, we can now define the EM approximate solution for (1). Given a step size $\Delta t>0$. Compute the discrete approximations $y_{k} \approx Y_{k \Delta t}$ by setting $y_{0}=Y_{0}, \widetilde{X_{0}}=X_{0}$ and

$$
y_{k+1}=y_{k}+a_{\widetilde{X_{k}}}\left(b_{\widetilde{X_{k}}}-y_{k}\right) \Delta t+\sigma_{\widetilde{X_{k}}} y_{k}^{\beta} \Delta W_{k},
$$

where $\Delta W_{k}=W_{(k+1) \Delta t}-W_{k \Delta t}$. Let

$$
\bar{y}(t)=y_{k}, \bar{x}(t)=\widetilde{X_{k}}, t \in[k \Delta t,(k+1) \Delta t), k=0,1,2, \cdots,
$$

and define the continuous EM approximate solution by

$$
y(t)=y_{0}+\int_{0}^{t} a_{\bar{x}(t)}\left(b_{\bar{x}(t)}-\bar{y}(t)\right) d t+\int_{0}^{t} \sigma_{\bar{x}(t)} \bar{y}(t)^{\beta} d W(t),
$$

Note that $Y_{k \Delta t}=y_{k}=\bar{y}_{k \Delta t}$, that is, $Y(t)$ and $\bar{y}(t)$ coincide with the discrete approximate solution at the grid points.

\section{CONVERGENCE OF THE EM APPROXIMATE SOLUTION}

The following theorem describes the convergence in probability of the EM solution to the exact solution under the local Lipschitz condition.

\section{A. Theorem 1}

For $Y(t)$ in (1) and $y(t)$ in (5),

$$
\lim _{\Delta t \rightarrow 0}\left(\sup _{0 \leq t \leq T}|Y(t)-y(t)|^{2}\right)=0 \text {, in probability. }
$$

\section{B. Proof.}

We divide the whole proof into three steps.

\section{Step 1}

For sufficiently large $R$, define the similar stopping time

$$
\tau_{R}=T \wedge \inf \{t \geq 0 \mid Y(t) \geq R\}
$$

Applying the generalized Ito formula to a $C^{2}$ function $V_{X_{t}}(y(t))$ yields

$$
\left.E V_{X\left(t \wedge \tau_{R}\right)}\left(Y\left(t \wedge \eta_{R}\right)\right)=V_{x_{0}}\left(y_{0}\right)+E \int_{0}^{t \wedge \tau_{R}} L V_{X(s)}(Y(s))\right] d s
$$

Using the Gronwall inequality, we obtain

$$
E V_{X\left(T \wedge \tau_{R}\right)}\left(Y\left(T \wedge \eta_{R}\right)\right) \leq e^{K T}\left[V_{x_{0}}\left(y_{0}\right)+K T\right]
$$

for some positive number $K$.

Let

$$
V_{R}=\inf \left\{V_{i}(Y(t)), Y(t) \geq R, i \in \Im\right\} .
$$

We can derive that

$$
P\left\{\tau_{R} \leq T\right\} \leq \frac{e^{K T}}{V_{R}}\left[V_{x_{0}}\left(y_{0}\right)+K T\right] .
$$

\section{Step 2}

For sufficiently large $R$, define the similar stopping time

$$
\eta_{R}=T \wedge \inf \{t \geq 0 \mid y(t) \geq R\}
$$

Using (5) and applying the generalized Ito's formula to $V_{X(t)}(y(t))$ defined in (2) yields

$$
\begin{gathered}
E V_{X\left(t \wedge \eta_{R}\right)}\left(y\left(t \wedge \eta_{R}\right)\right)=V_{x_{0}}\left(y_{0}\right) \\
+E \int_{0}^{t \wedge \eta_{R}}\left[a_{\bar{x}(s)}\left(b_{\bar{x}(s)}-\bar{y}(s)\right)\left(\frac{1}{2} \theta_{X(s)} y(s)^{-\frac{1}{2}}-2 \gamma_{X(s)} y(s)^{-3}\right)\right. \\
+\frac{1}{2} \sigma_{\bar{x}(s)}^{2} \bar{y}(s)^{2 \beta}\left(-\frac{1}{4} \theta_{X(s)} y(s)^{-\frac{2}{3}}+6 \gamma_{X(s)} y(s)^{-4}\right) \\
\left.+\sum_{j=1}^{G} q_{i j} V_{j}(y(s))\right] d s .
\end{gathered}
$$

Since $L V_{\bar{x}(s)}(\bar{y}(s)) \leq K\left[1+V_{\bar{x}(s)}(\bar{y}(s))\right]$, Rearranging the terms on the right-hand side by plus-and-minus technique we obtain that

$$
\begin{aligned}
& E V_{X\left(t \wedge \eta_{R}\right)}\left(y\left(t \wedge \eta_{R}\right)\right) \\
& \leq V_{x_{0}}\left(y_{0}\right)+K E \int_{0}^{t \wedge \eta_{R}}\left[1+V_{X(s)}(y(s))\right] d s \\
& +K E \int_{0}^{t \wedge \eta_{R}}\left[\theta_{\bar{X}(s)}(\sqrt{\bar{y}(s)}-\sqrt{y(s)})+\gamma_{\bar{x}(s)}\left(\bar{y}(s)^{-2}-y(s)^{-2}\right)\right] d s \\
& \left.+K E \int_{0}^{\star \wedge \eta_{R}}\left[\left(\theta_{\bar{x}(s)}-\theta_{X(s)}\right) \sqrt{y(s)}+\left(\gamma_{\bar{x}(s)}-\gamma_{X(s)}\right) y(s)^{-2}\right)\right] d s \\
& +E \int_{0}^{t \wedge \lambda_{R}} a_{\bar{x}(s)}\left(b_{\bar{x}(s)}-\bar{y}(s)\right)\left[\frac{1}{2} \theta_{X(s)}\left(y(s)^{-\frac{1}{2}}-\bar{y}(s)^{-\frac{1}{2}}\right)-2 \gamma_{X(s)}\left(y(s)^{-3}-\bar{y}(s)^{-3}\right)\right] d s \\
& +E \int_{0}^{t \wedge \eta_{R}} a_{\bar{x}(s)}\left(b_{\bar{x}(s)}-\bar{y}(s)\right)\left[\frac{1}{2}\left(\theta_{X(s)}-\theta_{\bar{x}(s)}\right) \bar{y}(s)^{-\frac{1}{2}}-2\left(\gamma_{X(s)}-\gamma_{\bar{x}(s)}\right) \bar{y}(s)^{-3}\right] d s \\
& +\frac{1}{2} E \int_{0}^{t \wedge \eta_{R}} \sigma_{\bar{x}(s)}^{2} \bar{y}(s)^{2 \beta}\left[-\frac{1}{4} \theta_{X(s)}\left(y(s)^{-\frac{2}{3}}-\bar{y}(s)^{-\frac{2}{3}}\right)+6 \gamma_{X(s)}\left(y(s)^{-4}-\bar{y}(s)^{-4}\right)\right] d s \\
& +\frac{1}{2} E \int_{0}^{t \wedge \eta_{R}} \sigma_{\bar{X}(s)}^{2} \bar{y}(s)^{2 \beta}\left[-\frac{1}{4}\left(\theta_{X(s)}-\theta_{\bar{X}(s)}\right) \bar{y}(s)^{-\frac{2}{3}}+6\left(\gamma_{X(s)}-\gamma_{\bar{X}(s)}\right) \bar{y}(s)^{-4}\right] d s \\
& +E \int_{0}^{t \wedge \eta_{R}} \sum_{j=1}^{G} q_{X(s) j}\left[\theta_{j}(\sqrt{y(s)}-\sqrt{\bar{y}(s)})+\gamma_{j}\left(y(s)^{-2}-\bar{y}(s)^{-2}\right)\right] d s \\
& +E \int_{0}^{t \wedge \eta_{R}} \sum_{j=1}^{G}\left(q_{X(s) j}-q_{\bar{x}(s) j}\right)\left(\theta_{j} \sqrt{\bar{y}(s)}+\gamma_{j} \bar{y}(s)^{-2}\right) d s .
\end{aligned}
$$

By condition (iii) we have 


$$
\begin{aligned}
E \int_{0}^{t \wedge \eta_{R}} & {\left[\theta_{\bar{x}(s)}(\sqrt{\bar{y}(s)}-\sqrt{y(s)})+\gamma_{\bar{x}(s)}\left(\bar{y}(s)^{-2}-y(s)^{-2}\right)\right] d s } \\
& \leq E \int_{0}^{t \wedge \eta_{R}}\left[\Theta|\sqrt{\bar{y}(s)}-\sqrt{y(s)}|+\Gamma\left|\bar{y}(s)^{-2}-y(s)^{-2}\right|\right] d s \\
& \leq C_{1}(R) E \int_{0}^{t \wedge \eta_{R}}|\bar{y}(s)-y(s)| d s \\
& \leq C_{1}(R) E \int_{0}^{T}\left|\bar{y}\left(s \wedge \eta_{R}\right)-y\left(s \wedge \eta_{R}\right)\right| d s \\
& \leq C_{1}(R) \int_{0}^{T}\left(E\left|\bar{y}\left(s \wedge \eta_{R}\right)-y\left(s \wedge \eta_{R}\right)\right|^{2}\right)^{\frac{1}{2}} d s
\end{aligned}
$$

Similarly, we can get

$$
\begin{aligned}
E \int_{0}^{t \wedge \eta_{R}} & a_{\bar{\Psi}(s)}\left(b_{\bar{\Psi}(s)}-\bar{y}(s)\right)\left[\frac{1}{2} \theta_{X(s)}\left(y(s)^{-\frac{1}{2}}-\bar{y}(s)^{-\frac{1}{2}}\right)-2 \gamma_{X(s)}\left(y(s)^{-3}-\bar{y}(s)^{-3}\right)\right] d s \\
& \leq A B E \int_{0}^{t \wedge \eta_{R}}\left[\frac{1}{2} \Theta\left|y(s)^{-\frac{1}{2}}-\bar{y}(s)^{-\frac{1}{2}}\right|-2 \Gamma\left|y(s)^{-3}-\bar{y}(s)^{-3}\right| d s\right. \\
& \leq C_{2}(R) E \int_{0}^{t \wedge \eta_{R}}|\bar{y}(s)-y(s)| d s \\
& \leq C_{2}(R) \int_{0}^{T}\left(E \mid \bar{y}\left(s \wedge \eta_{R}\right)-y\left(s \wedge \eta_{R}\right)^{2}\right)^{\frac{1}{2}} d s .
\end{aligned}
$$

We can show, in the same way as above, that

$$
\begin{aligned}
E \int_{0}^{t \wedge \eta_{R}} & {\left.\left[\left(\theta_{\bar{x}(s)}-\theta_{X(s)}\right) \sqrt{y(s)}+\left(\gamma_{\bar{x}(s)}-\gamma_{X(s)}\right) y(s)^{-2}\right)\right] d s } \\
& \left.\leq E \int_{0}^{T \wedge \eta_{R}}\left[2 \Theta \sqrt{y(s)}+2 \Gamma y(s)^{-2}\right)\right] I_{\{\bar{x}(s) \neq X(s)\}} d s \\
& \leq C_{2}(R) \sum_{k=0}^{N-1} \int_{t_{k}}^{t_{k+1}} E\left[I_{\left\{X\left(t_{k}\right) \neq X(s)\right\}} \mid X\left(t_{k}\right)\right] d s .
\end{aligned}
$$

Since

$$
\begin{aligned}
E\left[I_{\left\{X\left(t_{k}\right) \neq X(s)\right\}} \mid X\left(t_{k}\right)\right] & =\sum_{\substack{i=1 \\
G}} I_{\left\{X\left(t_{k}\right)=i\right\}} P\left(X(s) \neq i \mid X\left(t_{k}\right)=i\right) \\
& =\sum_{i=1}^{G} I_{\left\{X\left(t_{k}\right)=i\right\}} \sum_{j \neq i}\left(q_{i j}\left(s-t_{k}\right)+o\left(s-t_{k}\right)\right) \\
& \leq\left(\max _{1 \leq i \leq G}\left(-q_{i i}\right) \Delta t+o(\Delta t)\right) .
\end{aligned}
$$

Substituting this into (8) gives

$$
\begin{aligned}
\left.E \int_{0}^{t \wedge \eta_{R}}\left[\left(\theta_{\bar{x}(s)}-\theta_{X(s)}\right) \sqrt{y(s)}+\left(\gamma_{\bar{x}(s)}-\gamma_{X(s)}\right) y(s)^{-2}\right)\right] d s & \leq C_{2}(R) T\left(\max _{1 \leq i \leq G}\left(-q_{i}\right) \Delta t+o(\Delta t)\right) .
\end{aligned}
$$

We can similarly estimate the other terms on the right-hand side of (7) to get that

$$
\begin{aligned}
& E V_{X\left(t \wedge \eta_{R}\right)}\left(y\left(t \wedge \eta_{R}\right)\right) \\
& \quad \leq V_{x_{0}}\left(y_{0}\right)+K T+E \int_{0}^{t \wedge \eta_{R}} V_{X(s)}(y(s) d s \\
& \quad+C_{1}(R) \int_{0}^{T}\left(E\left|\bar{y}\left(s \wedge \eta_{R}\right)-y\left(s \wedge \eta_{R}\right)\right|^{2}\right)^{\frac{1}{2}} d s \\
& \quad+C_{1}(R) T\left(\max _{1 \leq i \leq G}\left(-q_{i i}\right) \Delta t+o(\Delta t)\right),
\end{aligned}
$$

where $C_{1}(R)$ is a cnstant dependent on $R$ but independent of $\Delta t$. Similarly we can show that

$$
E\left|\bar{y}\left(s \wedge \eta_{R}\right)-y\left(s \wedge \eta_{R}\right)\right|^{2} \leq C_{2}(R) \Delta t,
$$

Substituting this into (9) yields that

$$
\begin{aligned}
& E V_{X\left(t \wedge \eta_{R}\right)}\left(y\left(t \wedge \eta_{R}\right)\right) \\
& \quad \leq V_{x_{0}}\left(y_{0}\right)+K T+C_{3}(R)(\sqrt{\Delta t}+o(\Delta t))+K \int_{0}^{t} E V_{X(s)} y(s) d s,
\end{aligned}
$$

By the Gronwall inequality,

$$
E V_{X\left(T \wedge n_{R}\right)}\left(y\left(T \wedge \eta_{R}\right)\right) \leq e^{K T}\left[V_{x_{0}}\left(y_{0}\right)+K T+C_{3}(R)(\sqrt{\Delta t}+o(\Delta t))\right] .
$$

\section{E. $\quad$ Step 3}

In the same way as (6) was obtained, we can then show that

$$
P\left\{\eta_{R} \leq T\right\} \leq \frac{e^{K T}}{V_{R}}\left[V_{x_{0}}\left(y_{0}\right)+K T+C_{3}(R)(\sqrt{\Delta t}+o(\Delta t))\right] .
$$

Now, let $\varepsilon, \delta \in(0,1)$ be arbitrarily small. Set

$$
\Omega=\left\{\omega: \sup _{0 \leq t \leq T}|\bar{y}(t)-y(t)|^{2} \geq \delta\right\}
$$

We compute

$$
\begin{aligned}
P(\Omega)= & P(\Omega \cap\{\tau \geq T\})+P(\tau<T) \\
\leq & P(\Omega \cap\{\tau \geq T\})+P(\theta<T)+P(\rho<T) \\
\leq & \frac{C_{4}(R)}{\delta}(\Delta t+o(\Delta t))+\frac{2 e^{K T}}{V_{R}}\left[V_{x_{0}}\left(y_{0}\right)+K T\right] \\
& +\frac{e^{K T}}{V_{R}} C_{3}(R)(\sqrt{\Delta t}+o(\Delta t))
\end{aligned}
$$

Recalling that $V_{R} \rightarrow \infty$ as $R \rightarrow \infty$, we can choose $R$ sufficiently large for

$$
\frac{2 e^{K T}}{V_{R}}\left[V_{x_{0}}\left(y_{0}\right)+K T\right] \leq \frac{\varepsilon}{2}
$$

and then choose $\Delta t$ sufficiently small for

$$
\frac{C_{4}(R)}{\delta}(\Delta t+o(\Delta t))+\frac{e^{K T}}{V_{R}} C_{3}(R)(\sqrt{\Delta t}+o(\Delta t)) \leq \frac{\varepsilon}{2},
$$

to obtain 


$$
P(\Omega)=P\left\{\omega: \sup _{0 \leq t \leq T}|\bar{y}(t)-y(t)|^{2} \geq \delta\right\} \leq \varepsilon .
$$

This proves the assertion (5).

\section{CONCLUSION}

In this paper we obtain the Euler-Maruyama approximate solution for mean-reverting Regime Switching CEV processes and provides a detailed proof of the strong convergence of the EM approximate solution to the exact solution.

\section{ACKNOWLEDGMENT}

The authors would like to thank the Youth Project of National Social Sciences Foundation (No.12CGL021), the Youth Project in Humanities and Social Science Research of the Ministry of Education of China (No.11YJC790224), and the National Natural Science Foundation of China (No.71173203) for financial support.

\section{REFERENCES}

[1] F. Black, M. Scholes. "The pricing of options and corporate liabilities," Journal of Political Economy, vol 81,1973, pp. 637-659.

[2] R. Merton. "Theory of rational option pricing," Bell Journal of Economics and Management Science, vol 4,1973, pp. 141-183.

[3] J. Cox, "Notes on Option Pricing I: Constant Elasticity of Variance Diffusions,” Stanford university, Working paper, 1975.

[4] J. Cox, S. Ross. "The valuation of options for alternative stochastic processes,” Journal of Financial Economics, vol 3, 1976, pp. 145-166.

[5] Mendoza-Arriaga R, Linetsky V. "Pricing equity default swaps under the jump-to-default extended CEV model.” Finance and Stochastics, vol 15, 2011, pp. 513-540.

[6] Ruas J P, Dias J C, Nunes J P V. "Pricing and static hedging of American-style options under the jump to default extended CEV model.” Journal of Banking \& Finance, vol 37, 2013, pp. 4059-4072.

[7] Larguinho M, Dias J C, Braumann C A. "On the computation of option prices and Greeks under the CEV model.” Quantitative Finance, vol 13, 2013, pp. 907-917.

[8] Thakoor N, Tangman D Y, Bhuruth M. "A new fourth-order numerical scheme for option pricing under the CEV model." Applied Mathematics Letters, vol 26, 2013, pp. 160-164.

[9] Ang. A., Bekaert. G. "Regime switches in interest rates." Journal of Business and Economic Statistics, vol 20, 2012, pp. 163-182.

[10] Boyle. P., Draviam, T. "Pricing exotic options under regime switching.” Insurance: Mathematics and Economics, vol 40, 2007, pp. 267-282.

[11] Siu, T. K., Erlwein, C., Mamon, R. S. "The pricing of credit default swaps under a markov-modulated mertons structural model." North American Actuarial Journal,vol 12, 2008, pp. 18-46.

[12] Yao, D. D., Zhang, Q., Zhou, X. Y. A regime-switching model for European options. In Houmin Yan, Yin, G. G. and Zhang, Q. (Ed.), Stochastic processes, optimization, and control theory: applications in financial engineering, queueing networks, and manufacturing systems, 2006, pp. 281-300. Berlin: Springer.

[13] Maalaoui Chun, O., Dionne, G., Fran_cois, P. “Credit spread changes within switching regimes.” Journal of Financial and Quantitative Analysis, 2014, forthcoming.

[14] Schaller H, Norden SV. "Regime switching in stock market returns." Applied Financial Economics, vol 7, 1997, pp. 177-191.

[15] Ang A, Timmermann A. "Regime changes and financial markets." Annual Review of Financial Economics, vol 4, 2012, pp. 313-337. 